\title{
Alteration of enzymes and their application to nucleic acid amplification (Review)
}

\author{
KIYOSHI YASUKAWA ${ }^{1}$, ITARU YANAGIHARA ${ }^{2}$ and SHINSUKE FUJIWARA ${ }^{3}$ \\ ${ }^{1}$ Division of Food Science and Biotechnology, Graduate School of Agriculture, Kyoto University, Kyoto 606-8502;
${ }^{2}$ Department of Developmental Medicine, Research Institute, Osaka Women's and Children's Hospital, Izumi, Osaka 594-1101;
${ }^{3}$ Department of Bioscience, School of Science and Technology, Kwansei-Gakuin University, Sanda, Hyogo 669-1337, Japan
}

Received December 29, 2019; Accepted May 29, 2020

DOI: $10.3892 /$ ijmm.2020.4726

\begin{abstract}
Since the discovery of polymerase chain reaction (PCR) in 1985, several methods have been developed to achieve nucleic acid amplification, and are currently used in various fields including clinical diagnosis and life science research. Thus, a wealth of information has accumulated regarding nucleic acid-related enzymes. In this review, some nucleic acid-related enzymes were selected and the recent advances in their modification along with their application to nucleic acid amplification were described. The discussion also focused on optimization of the corresponding reaction conditions. Using newly developed enzymes under well-optimized reaction conditions, the sensitivity, specificity, and fidelity of nucleic acid tests can be improved successfully.
\end{abstract}

\section{Contents}

1. Introduction

2. Thermostabilization of reverse transcriptase

Correspondence to: Dr Kiyoshi Yasukawa, Division of Food Science and Biotechnology, Graduate School of Agriculture, Kyoto University, Oiwakecho, Kitashirakawa, Sakyo-Ku, Kyoto 606-8502, Japan

E-mail: yasukawa.kiyoshi.7v@kyoto-u.ac.jp

Abbreviations: AMV, avian myeloblastosis virus; DETECTR, DNA endonuclease-targeted CRISPR transreporter; HAD, helicase-dependent isothermal DNA amplification; HIV, human immunodeficiency virus; HPV, human papillomavirus; LAMP, loop-mediated isothermal amplification; MMLV, Moloney murine leukemia virus; NASBA, nucleic acid sequence-based amplification; NGS, next-generation sequencing; RCA, rolling circle amplification; RPA, recombinase polymerase amplification; RT, reverse transcriptase; SDA, strand displacement amplification; SHERLOCK, specific high sensitivity enzymatic reporter unlocking

Key words: cDNA synthesis, DNA polymerase, fidelity, helicase, $\mathrm{PCR}$, recombinase polymerase amplification, reverse transcriptase, sensitivity, specificity
3. Creation of the reverse transcriptase activity in thermostable DNA polymerase

4. Use of helicase to increase specificity

5. Fidelity evaluation with NGS

6. Use of recombinase and single-strand binding protein for isothermal DNA amplification

7. Other considerable factors involved in nucleic acid amplification

8. Conclusions and future perspectives

\section{Introduction}

Nucleic acid amplification tests are core technologies of clinical diagnosis. In pulmonary tuberculosis, such testing is capable of identifying Mycobacterium species in clinical respiratory samples more rapidly and accurately than sputum specimen examinations and culture-based methods. This advantage is key to appropriate treatment, prevention, and control of transmission of tuberculosis. In HIV detection, the nucleic acid amplification test is more sensitive and quantitative than other methods based on HIV-1-specific antibody or viral antigens, enabling the detection of HIV-1 at the initial stage of infection and the monitoring of disease progression $(1,2)$.

Various nucleic acid amplification technologies have been devised, but the most widely used is PCR. In basic research, most researchers use PCR primarily for amplification, possibly because primer design is convenient and the enzymes are available at a reasonable price (3). In clinical diagnosis, on the other hand, isothermal nucleic acid amplification methods such as nucleic acid sequence-based amplification (NASBA) (4), strand displacement amplification (SDA) (5), rolling circle amplification (RCA) (6), helicase-dependent isothermal DNA amplification (HAD) (7), and loop-mediated isothermal amplification (LAMP) (8) are also used. The advantage of isothermal amplifications over PCR is that they do not require a complex device such as thermal cycler, improving throughput in situations when large numbers of clinical samples must be processed, as well as facilitating point-of-care diagnosis (4-9).

The performance of a nucleic acid amplification test depends largely on the performance of the enzymes involved. Thermostable DNA polymerase, first identified in 
Thermus aquaticus (Taq) in 1976 (10), has become widely used since the discovery of PCR. Concerning performances of $\mathrm{Taq}$ polymerase, it was initially reported that the activity decreased to $50 \%$ at incubation at $95^{\circ} \mathrm{C}$ for $1.6 \mathrm{~h}$; the rate of processing was 60-150 nucleotides/sec; and the error rate was $0.38-1.32 \times 10^{4}$ errors/base (11). Since then, the performances of $\mathrm{Taq}$ polymerase were improved by genetic engineering. For example, the mutation of Phe667 into Tyr increased its efficiency of incorporation with ddNTP by $10^{3}$-fold (12), and fusion of the helix-hairpin-helix motifs of DNA topoisomerase $\mathrm{V}$ to $\mathrm{Taq}$ polymerase increased the enzyme's stability and processivity (13). The performances of DNA polymerases from the hyperthermophilic archaeon Thermococcus kodakarensis (KOD) or Pyrococcus furiosus (Pfu) and that from thermophilic bacteria Thermus thermophilus (Tth) have also been improved by genetic engineering. Today, they are widely used in PCR along with Taq polymerase.

In addition to altering the enzymes, it is also important to optimize the reaction conditions. In the amplification techniques using multiple enzymes, such as RT-PCR and NASBA, this process is more complicated because each enzyme has its own optimal condition. Another concern is lowering the risk of contamination. In this regard, it is preferable to perform one-tube reactions with real-time monitoring (14).

The aim of the review is to outline recent advances in nucleic acid amplification technologies. The foci of the study are, reverse transcriptase as an example of an enzyme that has been markedly improved by genetic engineering; recombination polymerase amplification, an isothermal amplification which has attracted a great deal of recent attention; and focus helicase, an enzyme which increases specificity and decreases noise in the amplification. Next-generation sequencing (NGS) was used to evaluate the fidelity of cDNA synthesis and the statistical method to optimize the reaction conditions.

\section{Thermostabilization of reverse transcriptase}

Reverse transcriptase (RT) has RNA- and DNA-dependent DNA polymerase and ribonuclease (RNase) $\mathrm{H}$ activities. It is responsible for RNA viral genome replication. Moloney murine leukemia virus (MMLV) RT and avian myeloblastosis virus (AMV) RT are widely used in cDNA synthesis (15) (Table I). MMLV RT is a $75-\mathrm{kDa}$ monomer, and AMV RT is a heterodimer consisting of an $\alpha$ subunit $(63-\mathrm{kDa})$ and a $\beta$ subunit $(95-k D a)(16,17)$. The result of the homology search performed using the search program DNA Data Bank of Japan (DDBJ; https://www.ddbj.nig.ac.jp/index-e.html) CLUSTALW and the crystal stuructures of MMLV RT is shown in Fig. 1. MMLV RT and the $\alpha$ subunit of AMV RT comprise the fingers, palm, thumb, connection, and RNase $\mathrm{H}$ domains. The $\beta$ subunit of AMV RT includes these five domains along with the C-terminal integrase domain. MMLV RT and AMV RT have two active sites. The active site for the DNA polymerase reaction is in the fingers/palm/thumb domain, and that for the $\mathrm{RNase} \mathrm{H}$ reaction is in the $\mathrm{RNase} \mathrm{H}$ domain.

Thermostability of DNA polymerases is important for their wide-range practical use. For cDNA synthesis, an elevated reaction temperature is highly desirable because it reduces RNA secondary structure and nonspecific binding of the primer. However, RT is thermolabile. The initial activities of MMLV RT and AMV RT are reduced by $50 \%$ at 44 and $47^{\circ} \mathrm{C}$, respectively, during a 10-min incubation (18). Thus, improving the thermostability of RT has been an important subject. The thermostabilities of MMLV RT (19-21) and AMV RT (20) were first improved by eliminating the RNase H activity. The thermostability of MMLV RT was improved by introducing the triple mutation E286R/E302K/L435R or E286R/E302K/L435R/D524A in which the negatively charged (Glu286 and Glu302) and hydrophobic (Leu435) residues that were thought to interact with a template-primer were replaced with positively charged residues, and the catalytic residue responsible for RNase H activity Asp524 was replaced with Ala (22). The thermostability of MMLV RT was also improved by the mutation of Val433 present on the molecular surface to Arg (23). Finally, a highly thermostable MMLV variant A32V/L72R/E286R/E302K/W388R/L435R was generated by combining the triple mutation E286R/E302K/L435R with the following mutations: The mutation of the internal residue, Ala32 to Val in order to stabilize the hydrophobic core, the mutation of the hydrophobic surface residue, Leu72 to Arg, and the mutation of Trp388 which is close to the negatively charged residues to Arg in order to introduce a salt bridge (24). In a random mutation assay followed by a combination of stabilizing mutations, E69K/E302R/W313F/L435G/N454K was generated using a filter assay (25), L139P/D200N/T330P/L603W/E607K was generated using emulsion PCR (26), and D200C was obtained by screening an amino acid scanning library (27). The amino acid residues mutated for thermostabilization are widespread throughout the molecule (Fig. 1B).

Recombinant MMLV RT is well expressed in the soluble fractions in Escherichia coli, from which sufficient amounts of active enzymes are purified. On the contrary, AMV RT has been barely expressed in the soluble fractions of E. coli. Instead, the active AMV RT $\alpha$ subunit was expressed in insect cells (28), and its thermostability was improved by introducing the triple mutation V238R/L388R/D450A, corresponding to E286R/W388R/D524A in MMLV RT (29). Notably, recombinant AMV RT has been successfully expressed in the soluble fractions in E. coli since then, and is now commercially available.

cDNA synthesis, as with PCR, is a key technology both in clinical diagnosis and basic research. However, cDNA synthesis is less sensitive than PCR. To circumvent this problem, a cDNA synthesis method using three enzymes, the thermostable MMLV RT quadruple variant E286R/E302K/W388R/D524A (described above), the genetically engineered family A DNA polymerase variant with RT activity from the hyperthermophile Thermotoga petrophila $\mathrm{K} 4\left(\mathrm{~K} 4 \mathrm{pol}_{\mathrm{L} 329 \mathrm{~A}}\right)$ which will be described in the next section and the DNA/RNA helicase from a hyperthermophilic archaeon Thermococcus kodakarensis (Tk-EshA), was developed (Table I). K4 pol $_{\mathrm{L} 329 \mathrm{~A}}$ and $T k$-EshA will be described later. In amplification techniques using multiple enzymes such as NASBA $(1,30)$, optimization is more complicated than when using a single enzyme as in the case of PCR. In this case, statistical methods such as Taguchi's method have been successfully used for optimization (31) (Fig. 2). The merit of statistical methods is that many factors can be optimized at the same time with the minimum number of experiments.

Stabilization of RT is desirable for cDNA synthesis. Improvement in the thermostability of MMLV RT and AMV 
Table I. Enzymes used for nucleic acid amplification.

\begin{tabular}{ll}
\hline Enzyme & \multicolumn{1}{c}{ Application } \\
\hline Reverse transcriptase (RT) & \\
AMV RT & cDNA synthesis, NASBA \\
MMLV RT & cDNA synthesis \\
DNA polymerase & \\
Taq polymerase & PCR \\
Tth polymerase & PCR, cDNA synthesis \\
K4pol L329A $^{\text {a }}$ & PCR, cDNA synthesis \\
RTX & PCR, cDNA synthesis \\
Bacillus subtilis polymerase & RPA \\
DNA helicase & \\
Tk-EshA & PCR, cDNA synthesis \\
Tk-Upf1 & PCR \\
Single-strand DNA-binding protein & \\
T4 gp32 & RPA \\
Recombinase & \\
T4 uvsY & RPA \\
T4 uvsX & RPA \\
\hline
\end{tabular}

${ }^{a}$ L329A variant of DNA polymerase from Thermotoga petrophila $\mathrm{K} 4,{ }^{\mathrm{b}} 16$-tuple variant of KOD DNA polymerase. KOD, Thermococcus kodakarensis; RPA, recombinase polymerase amplification.

RT is an important subject. Characterization of about 700 variants of phage T4 lysozyme revealed that there can be various kinds of effective stabilizing methods such as disulfide bridge, salt-bridge interaction, metal binding, and hydrophobic stabilization (32). We consider that the thermostabilities of MMLV RT and AMV RT may be further improved by combining stabilizing mutations.

\section{Creation of the reverse transcriptase activity in thermostable DNA polymerase}

The DNA-dependent DNA polymerase distinguishes suitable substrates DNA and dNTPs from unsuitable RNA and rNTPs. The exact mechanisms of this distinction are unknown, but two mechanisms have been proposed. One mechanism is for rNTP/dNTP distinction. In Klenow polymerase, the bulky 2 ' hydroxyl group of ribose interferes with the substrate-binding region of Klenow polymerase: Glu710 sterically blocks the 2 ' hydroxyl group of rNTP. As a result, the enzyme accepts dNTP but excludes rNTP (33). A similar hindrance effect was reported in archaeon Thermococcus litoralis family B DNA polymerase: Tyr412 exludes rNTP by acting as a steric gate for the $2^{\prime}$ hydroxyl group of ribose (34). The other mechanism is for template distinction. Archaeal family B DNA polymerase excludes uracil-containing templates, and DNA synthesis is prematurely arrested at the position where uracil is contained. By contrast, bacterial DNA polymerase I ignores the absence of the 5' methyl group in uracil, and accepts a uracil-containing template. Therefore, the 2 ' hydroxyl group of ribose is considered a key factor for the distinction of RNA/DNA for bacterial DNA-dependent DNA polymerase (35). A similar effect was reported in Klenow polymerase: Asn420 and Tyr423 in the 3'-5' exonuclease domain play a role in RNA exlusion by interfering with the $2^{\prime}$ hydroxyl group of the template molecule (36).

To generate thermostable RT using DNA polymerases from thermophilic bacteria and archaea, several approaches have been taken (37-42). Some bacterial DNA polymerases (Pol I) show reverse transcriptase activity in the presence of $\mathrm{Mn}^{2+}$. The Tth DNA polymerase from Thermus thermophilus also shows the RT activity $(37,38)$. It lacks a 3'-5' exonuclease domain, which contributes to fidelity in PCR. DNA polymerase I from the hyperthermophilic bacterium, Thermotoga $\mathrm{sp}$, possesses a 3'-5' exonuclease domain. A study on chimeric DNA polymerases from Thermotoga sp and Thermus sp showed that chimeric DNA polymerases with RT activity possessed attenuated 3'-5' exonuclease activity (42). Mutations were introduced into another DNA polymerase from Thermotoga petrophila K4 (K4PolI) to allow K4PolI to accept an RNA. Among the variants constructed, T326A, L329A, Q384A, F388A, M408A, and Y438A exhibited RT activity while their 3'-5' exonuclease activites were reduced. By contrast, $\mathrm{K} 4 \mathrm{Pol}_{\mathrm{N} 422 \mathrm{~A}}$ and $\mathrm{K} 4 \mathrm{Pol}_{\mathrm{F} 451 \mathrm{~A}}$ did not exhibit RT activity but possessed full 3'-5' exonuclease activity (43). These results suggest that there is a correlation between the gain of RT activity and the loss of 3'-5' exonuclease activity. On the other hand, introduction of random mutations into Taq polymerase showed that mutations in domains other than the 3'-5' exonuclease domain generated the mutants with RT activity (39). Further structural studies are needed to exlopre the mechnasim connecting RT and 3'-5' exonuclease activities.

Archaeal family B DNA polymerases, such as those from Pyrococcus furiosus (44) or Thermococcus kodakarensis (45), 
A

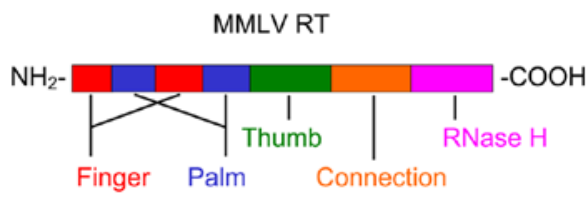

AMV RT

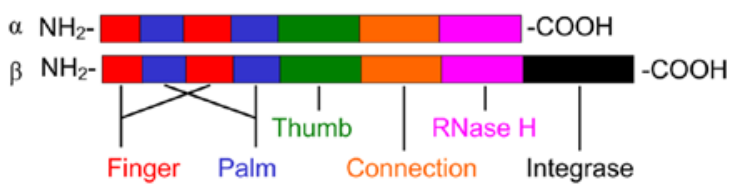

\section{B}

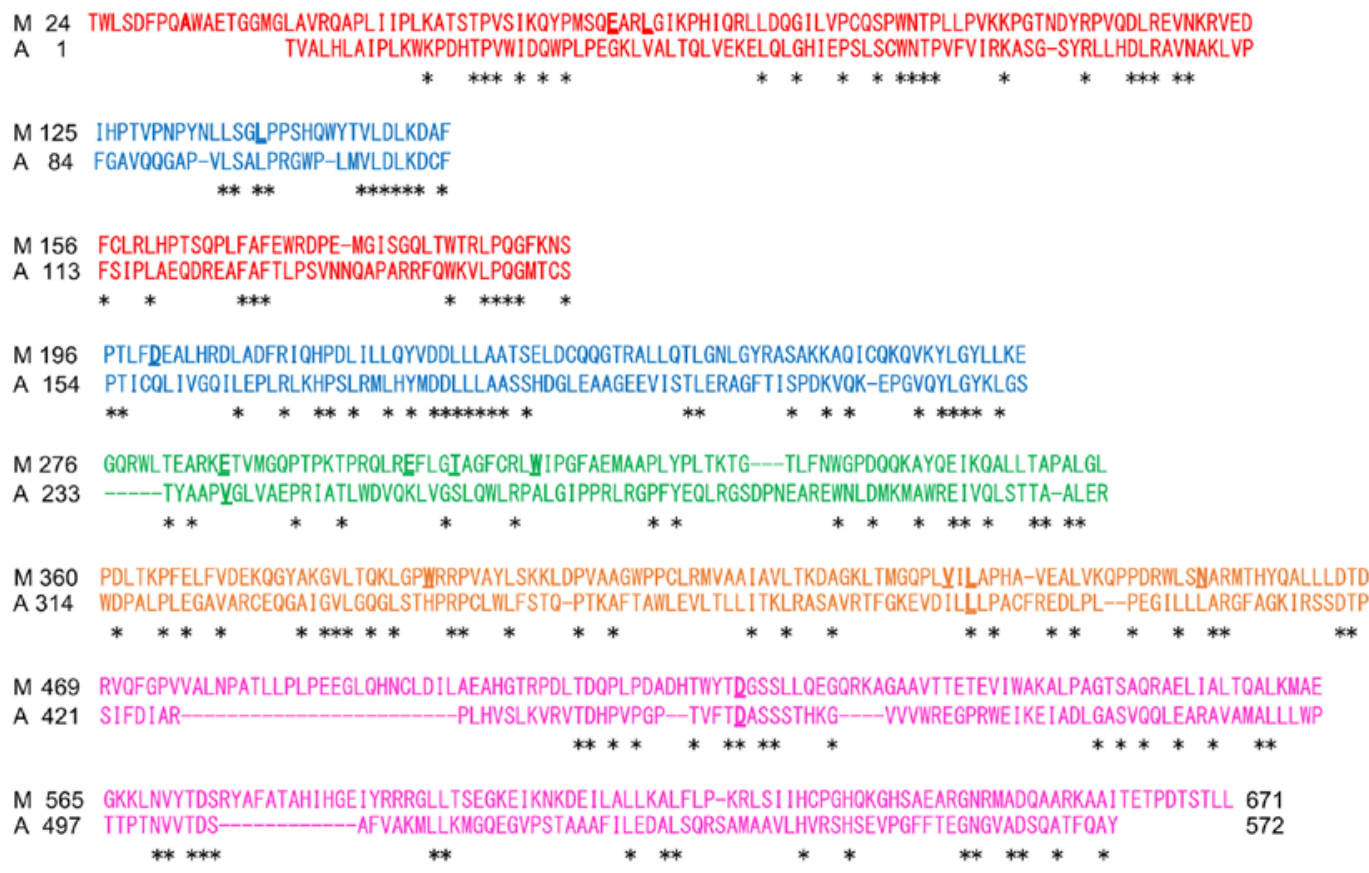

Figure 1. (A) Domain structures of MMLV RT and AMV RT. (B) A sequence alignment of MMLV RT and AMV RT. A homology search was performed using the search program DNA Data Bank of Japan (DDBJ) CLUSTALW and was revised based on the data of X-ray crystallographic analysis of MMLV RT. Asterisks show homologous amino acid residues. The amino acid residues to be mutated for thermostabilization are marked in bold and are underlined. Leu603 and Glu607 in ref. 26 correspond to Leu593 and Glu597, respectively.

possess a higher fidelity than thermophilic bacteria enzymes, such as those from T. aquaticus and T.thermophilus. However, as mentioned above, archaeal family B DNA polymerase excludes a template containing uracil, which is different from bacterial DNA polymerase (46). Family B DNA polymerase recognizes DNA more precisely than bacterial DNA polymerase I. Modified family B DNA polymerases with a Pol $\zeta$ fingers domain that displayed RT activity were developed by the mutation experiment into the $3^{\prime}-5^{\prime}$ exonuclease domain of hybrid archaeal family B DNA polymerases with a Pol $\zeta$ fingers domain (41). Recently, Ellefson et al generated a 16-tuple variant of KOD DNA polymerase known as RTX with RT activity from the hyperthermophilic archaeon, Thermococcus kodakarensis, by a directed evolution method (47). In this method, emulsion PCR was carried out with primers containing various numbers of ribonucleotides so that only DNA polymerase with RT activity enabled self-replication (47). These results indicate that family $B$
DNA polymerases can be used as a source to create reverse transcriptase.

DNA polymerases with RT activity enable one-stepRT-PCR without retroviral RT. The merit of one-step RT-PCR over two-step RT-PCR is that multiple openings of reaction tubes and reagent delivery are not necessary, leading to a decrease in DNA contamination risk. Furthermore, artificially created reverse transcriptase $\mathrm{K}_{4} \mathrm{pol}_{\mathrm{L} 329 \mathrm{~A}}$ and RTX are applicable for high sensitive RNA detection by one-step RT-PCR combining with the genetically engineered MMLV-RT and thermostable DNA/RNA helicase (48). COVID-19 RNA was also detected from clinical samples by using the system (data not shown). Details of the helicase role are mentioned below.

\section{Use of helicase to increase specificity}

DNA/RNA helicases exhibit nucleic acid binding, ATP hydrolysis, translocation, and unwinding of nucleic acid duplex by 


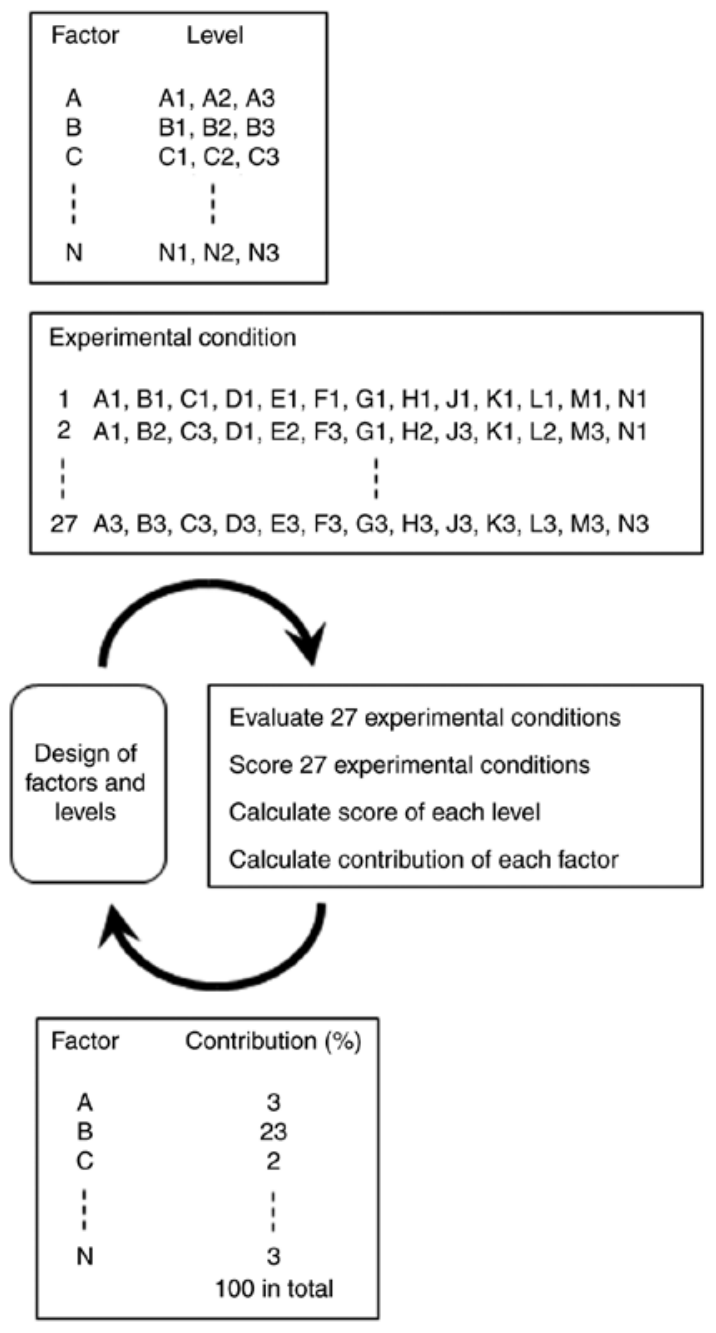

Figure 2. Application of Taguchi's method to optimize the reaction conditions with multiple enzymes. Schematic illustration of the cycle to optimize the reaction conditions is shown.

eliminating hydrogen bonds from the base-pairing between DNA/DNA, DNA/RNA, and RNA/RNA hybrids from the $3^{\prime}$ or $5^{\prime}$ unpaired end utilizing the energy generated upon ATP hydrolysis. Therefore, helicases are expected to unwind the secondary structured template and partially annealed primer/template duplexes in DNA and RNA synthesis. DNA/RNA helicases are classified into several superfamilies (SFs) according to their amino acid sequences (49). The SF1 and SF2 helicases are large and diverse groups, sharing catalytic cores with almost identical folds and extensive structural similarities. UvrD, an SF1 DNA helicase that unwinds blunt-end substrates as well as nicked circular DNA, was used in an isothermal DNA amplification at low temperature, called helicase-dependent amplification (50-53). In this amplification, a mesophilic DNA polymerase is applicable.

Unexpected DNAs sometimes get amplified due to primer mis-annealing during PCR. In order to efficiently reduce such mis-amplified products, an approach using helicase was devised (54). Tk-EshA, a euryarchaeota-specific SF2 helicase EshA from the hyperthermophilic archaeon Thermococcus kodakarensis, was first used for this purpose. In the presence of RNA, $T k$-EshA exhibited maximal ATPase activity at $80^{\circ} \mathrm{C}$. $T k$-EshA unwinds forked and $3^{\prime}$ overhung
DNAs (54). $T k$-EshA also possesses euryarchaeal termination activity (Eta), which disrupts the transcription elongation complex (55). We hypothesized that $T k$-EshA unwinds the structured template and peels off mis-annealed primers during PCR. To address this issue, PCR was performed using various DNAs as a substrate. When 16S rDNA was used, several mis-amplified products (noise DNAs) were detected in the absence of $T k$-EshA. However, they were eliminated in the presence of $T k$-EshA. These effects of $T k$-EshA were confirmed whether Taq DNA polymerase (a family A DNA polymerase, PolI type) or KOD DNA polymerase (a family B DNA polymerase, $\alpha$ type) was used. When toxA gene from Pseudomonas aeruginosa DNA, which possesses high GC content $(69 \%)$, was used, mis-amplified bands were also eliminated by the addition of $T k$-EshA, suggesting that $T k$-EshA was more effective than increasing the annealing temperature to reduce mis-amplified DNAs in the tox $A$ amplification (54). The action of $T k$-EshA is shown in Fig. 3. Another type (superfamily 1B) of helicase, Tk-Upf1 (TK0178) from T. kodakarensis, was examined for the effects on conventional PCR and digital PCR and compared with those of $T k$-EshA. It is important to eliminate nonspecific amplification for identification of SNPs. Of four double-stranded DNA substrates, forked, 5' overhung, 3' overhung, and blunt-ended DNAs, the unwinding activity of $T k$-Upf 1 was the highest towards 5' overhung DNAs (56). The concentration of $T k$-Upf1 required for noise DNA elimination was 10 -fold lower than that of $T k$-EshA. The addition of $T k$-Upf1 also eliminated noise DNAs derived from the misannealed primer when a $5^{\prime}$ or 3' overhung misannealed primer was included as a competitive primer along with specific primers. In digital PCR, Tk-EshA and $T k$-Upf1 functioned as signal enhancers: $T k$-EshA or $T k$-Upf1 increased the fluorescent intensities, improving separation between the common and risk allele clusters. The amount of $T k$-Upf1 required to improve the performance of digital PCR was smaller than that of $T k$-EshA.

\section{Fidelity evaluation with NGS}

Fidelity indicates the performance in the incorporation of correct nucleotides. Various methods have been applied to analyze DNA polymerase fidelity such as misincorporation (57), misextension (57), primer extension (58), and M13 lacZ mutation (59) assays. In a misincorporation assay, the reaction rates to incorporate correct and incorrect nucleotides are compared, while in a misextension assay, the reaction rates for extension from the mispaired end (i.e., A:G) and from the paired end (i.e., A:T) are compared (57). In these two assays, the reactions are carried out under single-turnover conditions. In a primer extension assay, the reaction in the absence of one dNTP is compared with that in the presence of all four dNTPs (58). In the M13 lacZ mutation assay, the error rates are calculated from the mutation frequency, which is determined as the ratio of mutant plaques to all plaques (59). The error rates of MMLV RT and AMV RT determined by this assay were $3.3-5.9 \times 10^{-4}$ errors/base and that of HIV-1 RT was $5.9 \times 10^{-3}$ errors/base (59). The M13 lacZ mutation assay has been the only method used to determine the error rate. However, it has some issues. Silent mutation affects the calculation of error rates. Identification of plaque color depends on 

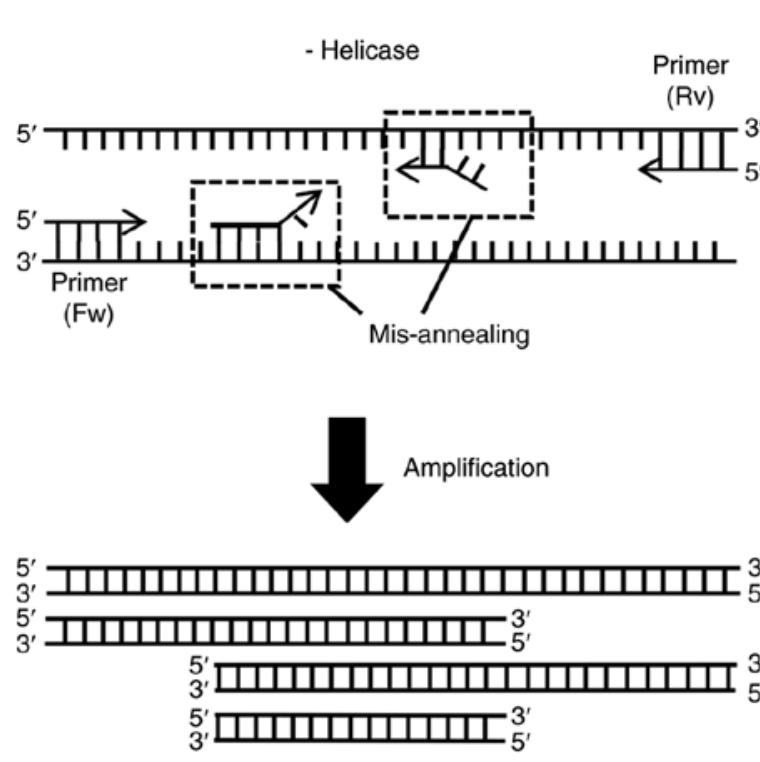

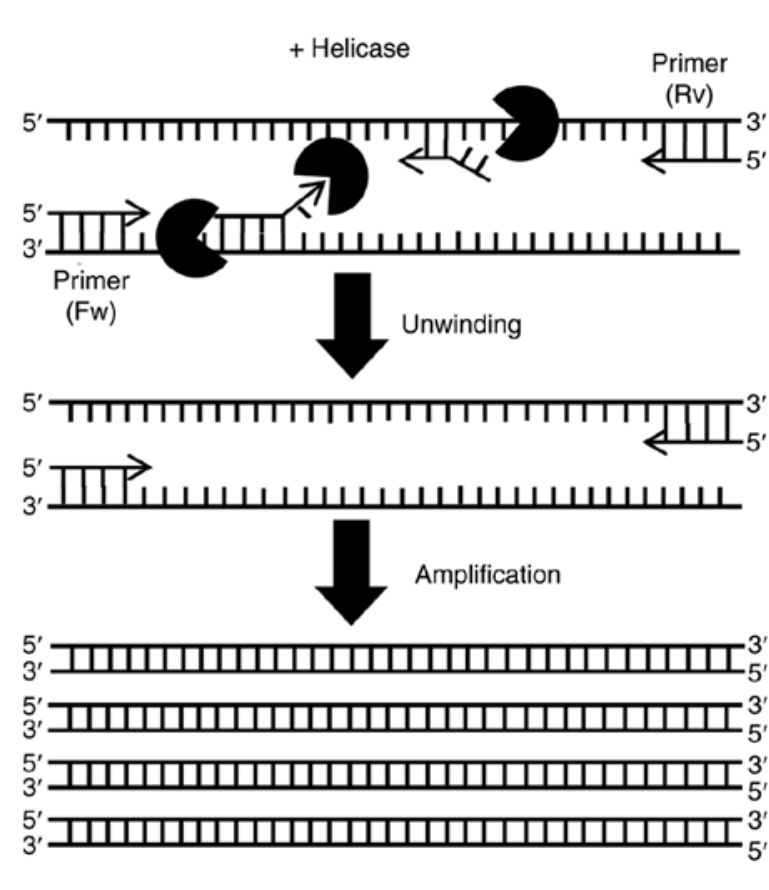

Figure 3. Model for noise reduction in PCR by helicase. Image of noise reduction in PCR by helicase such as a Tk-EshA is shown in the figure. In the absence of helicase, primers mis-anneal to various homologous regions, resulting in the amplification of noise DNAs. In the presence of helicase shown as a pacman, mis-annealed primers are peeled off and specific primers dominantly anneal to the target region, resulting in the reduced amplification of noise DNAs.

the individual. In addition, the reaction is DNA-dependent DNA synthesis, but not RNA-dependent DNA synthesis, even for RT.

In NGS, hundreds of million sequences are obtained in one NGS run. NGS has been widely used to identify rare mutations, misincorporations, and base modifications introduced in genomic DNA $(60,61)$. One of the problems of NGS is that a number of errors are introduced. To address this issue, a method to identify ultra-rare mutations in the genomic DNA using NGS was devised (62), which uses adaptors containing two tags of 12 randomized bases for the ligation of DNA fragments containing the sequences to be analyzed. All sequence reads are grouped based on tag sequences and orientations. By analyzing whether all sequence reads in the same group had the same mutation or not, each mutation that was observed via NGS indicated whether the error was already present in the genome or was incorporated by PCR or NGS (62).

We used NGS to determine the error rate of cDNA synthesis $(63,64)$. As shown in Fig. 4, cDNA was synthesized from a standard RNA with a primer possessing a tag of 14 randomized bases. All sequence reads are grouped based on tag sequences. By analyzing all sequence reads in the same group, each mutation revealed whether the error was incorporated by cDNA synthesis or not. The error rate obtained using this method of MMLV RT was $1.0 \times 10^{-4}$ errors/base and that with HIV-1 RT was $2.6 \times 10^{-4}$ errors/base (63), which was approximately $20 \%$ of those reported using the M13 lacZ mutation assay (59). Notably, unlike the M13 lacZ mutation assay, the NGS-based mutation assay reveals the mutation species and the frequency at each nucleotide position (63). This method may be effective in the assessment of the fidelity of various RTs with different reaction conditions: We reported that high concentrations of $\mathrm{dNTP}, \mathrm{MgCl}_{2}$, and $\mathrm{Mn}\left(\mathrm{OCOCH}_{3}\right)_{2}$ decreased the fidelity, and these effects were obvious in reactions using HIV-1 RT (64).

Fidelity of cDNA synthesis is important in clinical diagnosis and in life science research. The issue raised is how fidelity of RT and DNA polymerase can be ameliorated. One strategy is to optimize the concentrations of the enzyme, salts, and dNTP in the reaction solution. Another strategy is based on the studies conducted on HIV-1 RT (65-67). The fidelity of HIV-1 RT is lower than that of MMLV RT and AMV RT. One of the consequences of low fidelity of HIV-1 RT is the emergence of drug-resistant HIV-1 RT variants, such as K65R, R78A, and V75I. Interestingly, the mutations that confer drug resitance to these variants increase the fidelity of HIV-1 RT (65-67). This suggests that introduction of the corresponding mutations in MMLV RT or AMV RT may increase the fidelity, although such evidence has not yet been reported.

\section{Use of recombinase and single-strand binding protein for isothermal DNA amplification}

Recombinase polymerase amplification (RPA) is an isothermal reaction that is conducted at a temperature between 37 and $42^{\circ} \mathrm{C}$. RPA specifically amplifies a target DNA sequence with a recombinase, a single-stranded DNA-binding protein (SSB), and a strand-displacing polymerase (68). SSB binds to the primers and prevents oligonucleotide primers from forming secondary structures. Recombinase binds to the primers in the presence of ATP and with the assistance of the loading factor, T4 UvsY, which was originally identified as the T4 recombination mediator protein (69). The primers of the resulting complex bind to the homologous sequences of the DNA template using the ATP hydrolyzing activity of recombinase. In addition, SSB binds to the dispatched strand, 

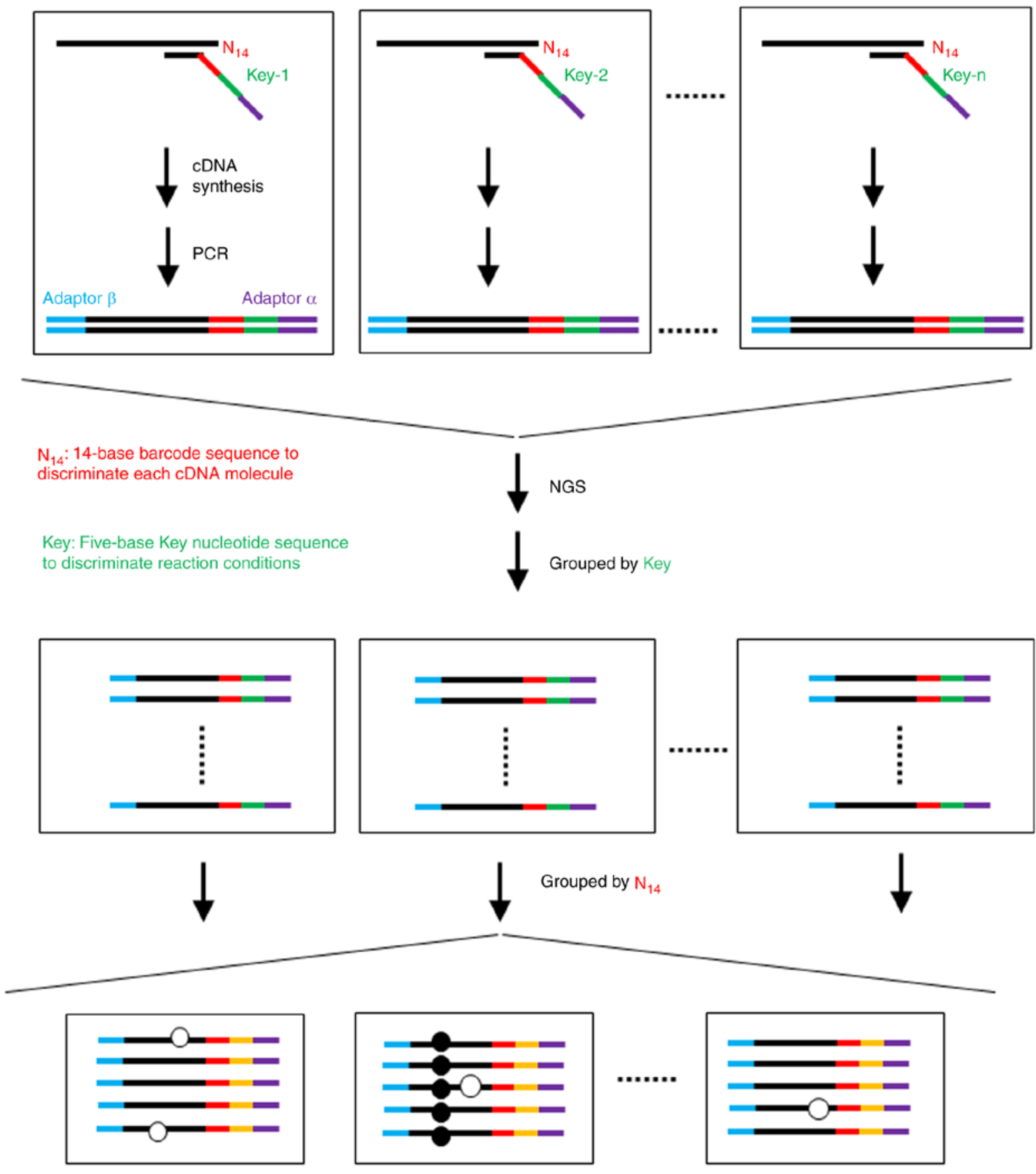

Grouped by $\mathrm{N}_{14}$
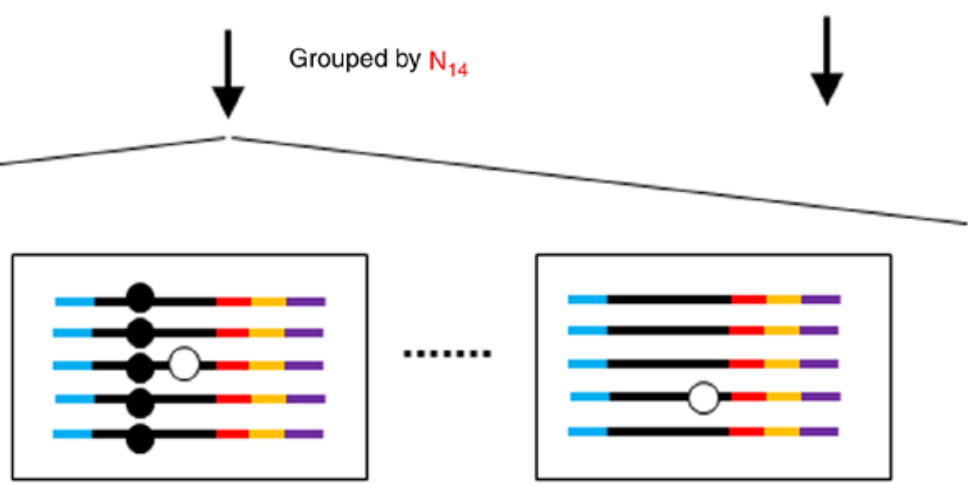

Error at cDNA synthesis

Error at PCR or NGS

Figure 4. Workflow of the analysis of the error rate in the cDNA synthesis reaction. $\mathrm{N}_{14}$, Key-n, and adaptor $\alpha$ and $\beta$ indicate the 14 -base randomized barcode sequence, five-base key nucleotide sequence, and Ion Proton sequencing adaptor $\alpha$ and $\beta$ sequences, respectively.

and strand-displacing polymerase extends the primer. Thus, the synthesis of a new DNA strand occurs (Fig. 5A).

In the first report of RPA in 2006 (70), T4 uvsX and T4 uvsY were used as recombinase, T4 gp32 was used as SSB, and Bacillus subtilis polymerase was used as strand-displacement DNA polymerase (Table I). Since then, RPA has been widely used to detect various targets. At present, the RPA kit is commercially available from TwistDX (Cambridge). One of the merits of RPA over other isothermal nucleic acid amplification methods is that the reaction occurs at the human body temperature $\left(37^{\circ} \mathrm{C}\right)$. RPA has the potential to eliminate the use of specialized equipment to provide the required temperature. Thus, RPA may be the most ideal nucleic acid amplification method for use in point-of-care diagnosis. Indeed, a number of RPA targets reported to date are pathogenic organisms including Mycobacterium tuberculosis $(71,72)$, Chlamydia trachomatis (73), Streptococcus pneumoniae (74), and Leishmania donovani (75).

In accordance with this trend, various technologies have been combined with RPA. For example, cutaneous 
A

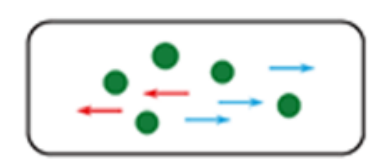

Recombinase

(T4 UvsX or E. coli RecA) and loading factor T4 UvsY
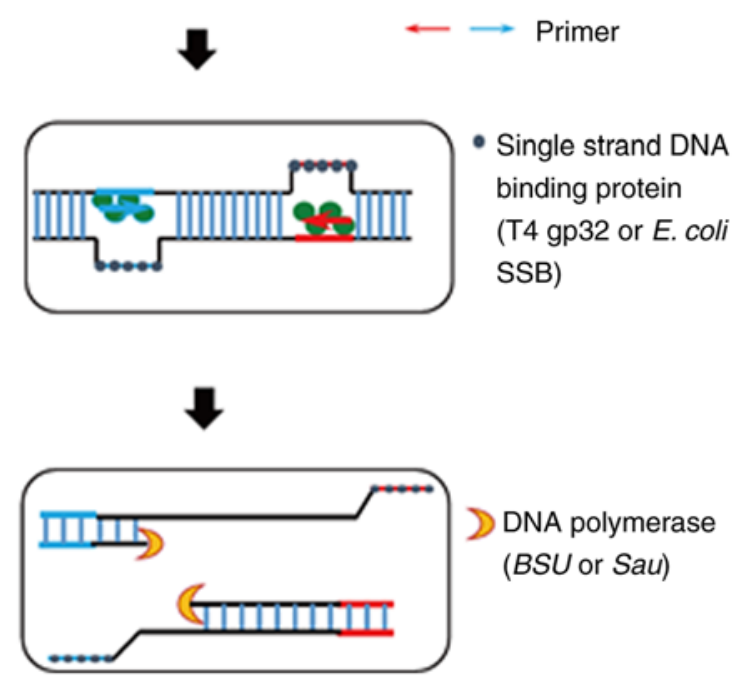

$\mathrm{B}$

$$
\text { SHERLOCK }
$$

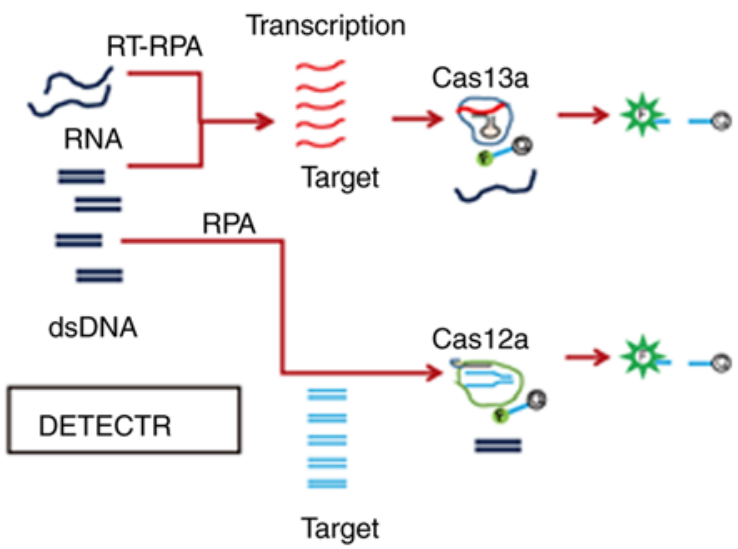

Figure 5. (A) Schematic illustration of the RPA process. (B) Models of SHERLOCK and DETECTR nucleic acid detection systems.

leishmaniasis was detected using an FTA card, a paper-based card commercialized by GE Healthcare for the isolation and storage of nucleic acids, and loop-mediated isothermal amplification (LAMP) (76,77). Lateral flow assay (78), enzyme-linked oligonucleotide assay (79), and electrochemical method (80) were used for end-point detection of RPA amplicons, whereas solid phase amplification was used for the real-time detection of RPA amplicons (81).

Clustered, regularly interspaced, short, palindromic repeats (CRISPR)/CRISPR-associated (CAS) systems were originally identified as an RNA-guided genetic silencing system in bacteria and archaea (82). At present, CRISPR/CAS9 is widely used in genome engineering. CRISPR-Cas13a and CRISPR-Cas12a have been applied to RPA (Fig. 5B). Specific high sensitivity enzymatic reporter unlocking (SHERLOCK) was established using Cas13a, an RNA-guided RNase that cleaves its specific target as well as the nearby non-targeted RNAs (collateral effect). The collateral cleavage enables release of the quenched fluorescent reporter (83). A multiplexed detection system was also established using Cas13, Cas12a, and Csm6 (84). Use of SHERLOCK allowed detection of Zika virus (sensitivity $2 \mathrm{aM}$ ) and that of a single nucleotide polymorphism of a human gene $(83,84)$. DNA endonuclease-targeted CRISPR transreporter (DETECTR) was established using CAS12a, an RNA-guided DNase. The DETECTR detected human papillomavirus (HPV) 16 and 18 at attomolar levels (85). These approaches may thus serve as valuable tools to increase the sensitivity of RPA and provide a means for developing novel point-of-care diagnosis with high sensitivity and rapidness.

\section{Other considerable factors involved in nucleic acid amplification}

Various factors are known to be involved in enzymatic reactions, and such factors include organic solvents. Enzymes are generally inactivated by organic solvents, but use of organic additives in enzymatic reactions can sometimes make previously problematic processes feasible. Indeed, various organic additives have been used to improve reaction efficiency and specificity in PCR $(86,87)$. Dimethyl sulfoxide (DMSO) and formamide have been used to improve specificity for the reaction with a $\mathrm{G}+\mathrm{C}$-rich DNA $(88,89)$. In cDNA synthesis, DMSO and formamide increased the reaction efficiency to some extent (90).

Since nucleic acids are highly negatively charged, they may be affected by positively charged small molecules such as polyamines. It was initially reported that spermidine was not beneficial in PCR (91). However, subsequent reports showed that spermidine prevents PCR inhibition problems encountered while analyzing clinical stool samples $(92,93)$. By optimizing the effects of these polar molecules, the efficiency of nucleic acid amplification is expected to further improve.

\section{Conclusions and future perspectives}

Despite being a widespread analytical method both in fundamental research and clinical diagnosis, there are limitations in nucleic acid amplification, which are represented by false-positive and false-negative results. Many efforts are still being devoted to improve the sensitivity, specificity, rapidness, and accuracy of nucleic acid amplification. The catalytic mechanism of nucleic acid-related enzymes has been extensively investigated by means of X-ray crystallography, kinetic analysis, and site-directed mutagenesis, leading to the generation of enzymes exhibiting extremely high activity and stability. Such enzymes and optimized reaction conditions offer many advantages that can be expected to enhance the efficiency of nucleic acid amplification tests, which may meet the increasing demand of point-of-care diagnosis both in developed and developing countries. 


\section{Acknowledgements}

We would like to thank Dr Kenji Kojima, Division of Food Science and Biotechnology, Graduate School of Agriculture, Kyoto University for insightful comments.

\section{Funding}

This review was supported by SENTAN (K.Y., I.Y., S.F.) from Japan Science and Technology Agency, Grants-in-Aid for Scientific Research (grant nos. 21580110, 18K19839 and 18K19839 for K.Y.) from Japan Society for the Promotion of Science, Emerging/re-emerging infectious disease project of Japan (K.Y., I.Y., S.F.) from Japan Agency for Medical Research and Development (grant no. JP20fk0108143 for K.Y., I.Y., S.F.) and Grant Program for Biomedical Engineering Research (K.Y., I.Y., S.F.) from Nakatani Foundation, Japan.

\section{Availability of data and materials}

Not applicable.

\section{Authors' contributions}

KY, IY and SF contributed to conceiving and designing the study, drafted and wrote the manuscript. All authors have read and approved the final version ofthe manuscript.

\section{Ethics approval and consent to participate}

Not applicable.

\section{Patient consent for publication}

Not applicable.

\section{Competing interests}

The authors declare that they have not competing interests.

\section{References}

1. Seki M, Kim CK, Hayakawa S and Mitarai S: Recent advances in tuberculosis diagnostics in resource-limited settings. Eur J Clin Microbiol Infect Dis 37: 1405-1410, 2018.

2. Zhao J, Chang L and Wang L: Nucleic acid testing and molecular characterization of HIV infections. Eur J Clin Microbiol Infect Dis 38: 829-842, 2019.

3. Mullis KB and Faloona FA: Specific synthesis of DNA in vitro via a polymerase-catalyzed chain reaction. Methods Enzymol 155: 335-350, 1987.

4. Kievits T, van Gemen B, van Strijp D, Schkkink P, Dircks M, Adriaanse H, Malek L, Sooknanan R and Lens P: NASBA isothermal enzymatic in vitro nucleic acid amplification optimized for the diagnosis of HIV-1 infection. J Virol Methods 35: 273-286, 1991

5. Walker GT, Fraiser MS, Schram JL, Little MC, Nadeau JG and Malinowski DP: Strand displacement amplification-an isothermal, in vitro DNA amplification technique. Nucleic Acids Res 20: 1691-1696, 1992.

6. Lizardi PM, Huang X, Zhu Z, Bray-Ward P, Thomas DC and Ward DC: Mutation detection and single-molecule counting using isothermal rolling-circle amplification. Nat Genet 19: 225-232, 1998

7. Vincent $\mathrm{M}, \mathrm{Xu} \mathrm{Y}$ and Kong $\mathrm{H}$ : Helicase-dependent isothermal DNA amplification. EMBO Rep 5: 795-800, 2004.
8. Notomi T, Okayama H, Masubuchi H, Yonekawa T, Watanabe K, Amino $\mathrm{N}$ and Hase T: Loop-mediated isothermal amplification of DNA. Nucleic Acids Res 28: E63, 2000.

9. Yan L, Zhou J, Zheng Y, Gamson AS, Roembke BT, Nakayama S and Sintim HO: Isothermal amplified detection of DNA and RNA. Mol Biosyst 10: 970-1003, 2014.

10. Chien A, Edgar DB and Trela JM: Deoxyribonucleic acid polymerase from the extreme thermophile Thermus aquaticus. J Bacteriol 127: 1550-1557, 1976.

11. Pavlov AR, Pavlova NV, Kozyavkin SA and Slesarev AI: Recent developments in the optimization of thermostable DNA polymerases for efficient applications. Trends Biotechnol 22: 253-260, 2004.

12. Tabor S and Richardson CC: A single residue in DNA polymerases of the Escherichia coli DNA polymerase I family is critical for distinguishing between deoxy- and dideoxyribonucleotides. Proc Natl Acad Sci USA 92: 6339-6343, 1995.

13. Pavlov AR, Belova GI, Kozyavkin SA and Slesarev AI: Helix-hairpin-helix motifs confer salt resistance and processivity on chimeric DNA polymerases. Proc Natl Acad Sci USA 99: 13510-13515, 2002.

14. Mallet F, Oriol G, Mary C, Verrier B and Mandrand B: Continuous RT-PCR using AMV-RT and Taq DNA polymerase: Characterization and comparison to uncoupled procedures. Biotechniques 18: 678-687, 1995.

15. Kimmel AR and Berger SL: Preparation of cDNA and the generation of cDNA libraries: Overview. Methods Enzymol 152: 307-316, 1987.

16. Georgiadis MM, Jessen SM, Ogata CM, Telesnitsky A, Goff SP and Hendrickson WA: Mechanistic implications from the structure of a catalytic fragment of Moloney murine leukemia virus reverse transcriptase. Structure 3: 879-892, 1995.

17. Das D and Georgiadis MM: The crystal structure of the monomeric reverse transcriptase from Moloney murine leukemia virus. Structure 12: 819-829, 2004.

18. Yasukawa K, Nemoto D and Inouye K: Comparison of the thermal stabilities of reverse transcriptases from avian myeloblastosis virus and Moloney murine leukaemia virus. J Biochem 143: 261-268, 2008.

19. Kotewicz ML, Sampson CM, D'Alessio JM and Gerard GF: Isolation of cloned Moloney murine leukemia virus reverse transcriptase lacking ribonuclease $\mathrm{H}$ activity. Nucleic Acids Res 16: 265-277, 1988.

20. Gerard GF, Potter RJ, Smith MD, Rosenthal K, Dhariwal G, Lee $\mathrm{J}$ and Chatterjee DK: The role of template-primer in protection of reverse transcriptase from thermal inactivation. Nucleic Acids Res 30: 3118-3129, 2002.

21. Mizuno M, Yasukawa K and Inouye K: Insight into the mechanism of the stabilization of Moloney murine leukaemia virus reverse transcriptase by eliminating RNase $\mathrm{H}$ activity. Biosci Biotechnol Biochem 74: 440-442, 2010.

22. Yasukawa K, Mizuno M, Konishi A and Inouye K: Increase in thermal stability of Moloney murine leukaemia virus reverse transcriptase by site-directed mutagenesis. J Biotechnol 150: 299-306, 2010.

23. Konishi A, Ma X and Yasukawa K: Stabilization of Moloney murine leukemia virus reverse transcriptase by site-directed mutagenesis of the surface residue Val433. Biosci Biotechnol Biochem 78: 147-150, 2014.

24. Baba M, Kakue R, Leucht C, Rasor P, Walch H, Ladiges D, Bell C, Kojima K, Takita T and Yasukawa K: Further increase in thermostability of Moloney murine leukemia virus reverse transcriptase by mutational combination. Protein Eng Des Sel 30: 551-557, 2017

25. Arezi B and Hogrefe H: Novel mutations in Moloney murine leukemia virus reverse transcriptase increase thermostability through tighter binding to template-primer. Nucleic Acids Res 37: 473-481 2009.

26. Baranauskas A, Paliksa S, Alzbutas G, Vaitkevicius M,Lubiene J, Letukiene V, Burinskas S, Sasnauskas G and Skirgaila R: Generation and characterization of new highly thermostable and processive M-MuLV reverse transcriptase variants. Protein Eng Des Sel 25: 657-668, 2012.

27. Katano Y, Li T, Baba M, Nakamura M, Ito M, Kojima K, Takita T and Yasukawa K: Generation of thermostable Moloney murine leukemia virus reverse transcriptase variants using site saturation mutagenesis library and cell-free protein expression system. Biosci Biotechnol Biochem 81: 2339-2345, 2017.

28. Konishi A, Nemoto D, Yasukawa K and Inouye K: Comparison of the thermal stabilities of the $\alpha \beta$ heterodimer and the $\alpha$ subunit of avian myeloblastosis virus reverse transcriptase. Biosci Biotechnol Biochem 75: 1618-1620, 2011. 
29. Konishi A, Yasukawa $\mathrm{K}$ and Inouye K: Improving the thermal stability of avian myeloblastosis virus reverse transcriptase $\alpha$-subunit by site-directed mutagenesis. Biotechnol Lett 34: 1209-1215, 2012.

30. Yasukawa K, Agata N and Inouye K: Detection of cesA mRNA from Bacillus cereus by RNA-specific amplification. Enzyme Microb Technol 46: 391-396, 2009.

31. Okano H, Katano Y, Baba M, Fujiwara A, Hidese R, Fujiwara S, Yanagihara I, Hayashi T, Kojima K, Takita T and Yasukawa K: Enhanced detection of RNA by MMLV reverse transcriptase coupled with thermostable DNA polymerase and DNA/RNA helicase. Enzyme Microb Technol 96: 111-120, 2017.

32. Baase WA, Liu L, Tronrud DF and Matthews BW: Lessons from the lysozyme of phage T4. Protein Sci 19: 631-641, 2010.

33. Astatke M, Ng K, Grindley ND and Joyce CM: A single side-chain prevents Escherichia coli DNA polymerase I (Klenow fragment) from incorporating ribonucleotides. Proc Natl Acad Sci USA 95: 3402-3407, 1998.

34. Gardner AF and Jack WE: Determinants of nucleotide sugar recognition in an archaeon DNA polymerase. Nucleic Acids Res 27: 2545-2553, 1999

35. Lin TC, Wang CX, Joyce CM and Konigsberg WH: 3'-5 Exonucleolytic activity of DNA polymerases: Structural features that allow kinetic discrimination between ribo- and deoxyribonucleotide residues. Biochemistry 40: 8749-8755, 2001.

36. Lam WC, Thompson EH, Potapova O, Sun XC, Joyce CM and Millar DP: 3'-5' Exonuclease of Klenow fragment: Role of amino acid residues within the single-stranded DNA binding region in exonucleolysis and duplex DNA melting. Biochemistry 41 : 3943-3951, 2002.

37. Shandilya H, Griffiths K, Flynn EK, Astatke M, Shih PJ, Lee JE, Gerard GF, Gibbs MD and Bergquist PL: Thermophilic bacterial DNA polymerases with reverse-transcriptase activity. Extremophiles 8: 243-251, 2004.

38. Myers TW and Gelfand DH: Reverse transcription and DNA amplification by a Thermus thermophilus DNA polymerase. Biochemistry 30: 7661-7666, 1991.

39. Ong JL, Loakes D, Jaroslawski S, Too K and Holliger P: Directed evolution of DNA polymerase, RNA polymerase and reverse transcriptase activity in a single polypeptide. J Mol Biol 361: 537-550, 2006.

40. Kranaster R, Drum M, Engel N, Weidmann M, Hufert FT and Marx A: One-step RNA pathogen detection with reverse transcriptase activity of a mutated thermostable Thermus aquaticus DNA polymerase. Biotechnol J 5: 224-231, 2010.

41. Jozwiakowski SK and Connolly BA: A modified family-B archaeal DNA polymerase with reverse transcriptase activity. Chembiochem 12: 35-37, 2011.

42. Schönbrunner NJ, Fiss EH, Budker O, Stoffel S, Sigua CL, Gelfand DH and Myers TW: Chimeric thermostable DNA polymerases with reverse transcriptase and attenuated 3'-5 exonuclease activity. Biochemistry 45: 12786-12795, 2006.

43. Sano S, Yamada Y, Shinkawa T, Kato S, Okada T, Higashibata $H$ and Fujiwara S: Mutations to create thermostable reverse transcriptase with bacterial family A DNA polymerase from Thermotoga petrophila K4. J Biosci Bioeng 113: 315-321, 2012.

44. Cline J, Braman JC and Hogrefe HH: PCR fidelity of pfu DNA polymerase and other thermostable DNA polymerases. Nucleic Acids Res 24: 3546-3551, 1996.

45. Takagi M, Nishioka M, Kakihara H, Kitabayashi M, Inoue H, Kawakami B, Oka M and Imanaka T: Characterization of DNA polymerase from Pyrococcus sp. strain KOD1 and its application to PCR. Appl Environ Microbiol 63: 4504-4510, 1997.

46. Firbank SJ, Wardle J, Heslop P, Lewis RJ and Connolly BA Uracil recognition in archaeal DNA polymerases captured by X-ray crystallography. J Mol Biol 381: 529-539, 2008.

47. Ellefson JW, Gollihar J, Shroff R, Shivram H, Iyer VR and Ellington AD: Synthetic evolutionary origin of a proofreading reverse transcriptase. Science 352: 1590-1593, 2016.

48. Okano H, Baba M, Kawato K, Hidese R, Yanagihara I, Kojima K, Takita T, Fujiwara S and Yasukawa K: High sensitive RNA detection by one-step RT-PCR using the genetically engineered variant of DNA polymerase with reverse transcriptase activity from hyperthermophilies. J Biosci Bioeng 125: 275-281, 2018.

49. Singleton MR, Dillingham MS and Wigley DB: Structure and mechanism of helicases and nucleic acid translocases. Annu Rev Biochem 76: 23-50, 2007.

50. An L, Tang W, Ranalli TA, Kim HJ, Wytiaz J and Kong H: Characterization of a thermostable UvrD helicase and its participation in helicase-dependent amplification. J Biol Chem 280: 28952-28958, 2005
51. Jeong YJ, Park K and Kim DE: Isothermal DNA amplification in vitro: The helicase-dependent amplification system. Cell Mol Life Sci 66: 3325-3336, 2009.

52. Artiushin S, Tong Y, Timoney J, Lemieux B, Schlegel A and Kong H: Thermophilic helicase-dependent DNA amplification using the IsoAmp ${ }^{\mathrm{TM}} \mathrm{SE}$ experimental kit for rapid detection of Streptococcus equi subspecies equi in clinical samples. J Vet Diagn Invest 23: 909-914, 2011.

53. Runyon GT and Lohman TM: Escherichia coli helicase II (UvrD) protein can completely unwind fully duplex linear and nicked circular DNA. J Biol Chem 264: 17502-17512, 1989.

54. Fujiwara A, Kawato K, Kato S, Yasukawa K, Hides R and Fujiwara S: Application for a euryarchaeota-specific helicase from Thermococcus kodakarensis and its application for noise reduction in PCR. Appl Environ Microbiol 82: 3022-3031, 2016.

55. Walker JE, Luyties $O$ and Santangelo TJ: Factor-dependent archaeal transcription termination. Proc Natl Acad Sci USA 114: E6767-E6773, 2017.

56. Hidese R, Kawato K, Nakura Y, Fujiwara A, Yasukawa K, Yanagihara I and Fujiwara S: Thermostable DNA helicase improves the sensitivity of digital PCR. Biochem Biophys Res Commun 495: 2189-2194, 2018.

57. Gutiérrez-Rivas M, Ibáñez Á, Martínez MA, Domingo E and Menéndez-Arias L: Mutational analysis of Phe160 within the 'palm' subdomain of human immunodeficiency virus type 1 reverse transcriptase. J Mol Biol 290: 615-625, 1999.

58. Kati WM, Johnson KA, Jerva LF and Anderson KS: Mechanism and fidelity of HIV reverse transcriptase. J Biol Chem 267: 25988-25997, 1992.

59. Bebenek K and Kunkel TA: Analyzing fidelity of DNA polymerase. Methods Enzymol 262: 217-232, 1995.

60. Shendure J and Ji H: Next-generation DNA sequencing. Nat Biotechnol 26: 1135-1145, 2008.

61. Iida $\mathrm{K}$, Jin $\mathrm{H}$ and Zhu JK: Bioinformatics analysis suggests base modifications of tRNAs and miRNAs in Arabidopsis thaliana. BMC Genomics 10: 155, 2009.

62. Schmitt MW, Kennedy SR, Salk JJ, Fox EJ, Hiatt JB and Loeb LA: Detection of ultra-rare mutations by next-generation sequencing. Proc Natl Acad Sci USA 109: 14508-14513, 2012.

63. Yasukawa K, Iida K, Okano H, Hidese R, Baba M, Yanagihara I, Kojima K, Takita $\mathrm{T}$ and Fujiwara S: Next-generation sequencing-based analysis of reverse transcriptase fidelity. Biochem Biophys Res Commun 492: 147-153, 2017.

64. Okano H, Baba M, Hidese R, Iida K, Li T, Kojima K, Takita T, Yanagihara I, Fujiwara S and Yasukawa K: Accurate fidelity analysis of the reverse transcriptase by a modified next-generation sequencing. Enzyme Microb Technol 115: 81-85, 2018.

65. Barrioluengo V, Álvarez M, Barbieri D and Menéndez-Arias L: Thermostable HIV-1 group O reverse transcriptase variants with the same fidelity as murine leukaemia virus reverse transcritpase. Biochem J 436: 599-607, 2011.

66. Álvarez M, Barrioluengo V, Afonso-Lehmann RN and Menéndez-Arias L: Altered error specificity of RNase H-deficient HIV-1 reverse transcriptases during DNA-dependent DNA synthesis. Nucleic Acid Res 41: 4601-4612, 2013.

67. Garforth SJ, Domaoal RA, Lwatula C, Landau MJ, Meyer AJ, Anderson KS and Prasad VR: K65R and K65A substitutions in HIV-1 reverse transcriptase enhance polymerase fidelity by decreasing both dNTP misinsertion and mispaired primer extension efficiencies. J Mol Biol 401: 33-44, 2010.

68. Li J, Macdonald J and von Stetten F: Review: A comprehensive summary of a decade development of the recombinase polymerase amplification. Analyst 144: 31-67, 2018.

69. Bleuit JS, Xu H, Ma Y, Wang T, Liu J and Morrical SW: Mediator proteins orchestrate enzyme-ssDNA assembly during T4 recombination-dependent DNA replication and repair. Proc Natl Acad Sci USA 98: 8298-8305, 2001.

70. Piepenburg O, Williams CH, Stemple DL and Armes NA: DNA detection using recombination proteins. PLoS Biol 4: e204, 2006.

71. Boyle DS, McNerneyR, Teng Low H,LeaderBT,Pérez-Osorio AC, Meyer JC, O'Sullivan DM, Brooks DG, Piepenburg O and Forrest MS: Rapid detection of Mycobacterium tuberculosis by recombinase polymerase amplification. PLoS One 9: e103091, 2014.

72. Shin Y, Perera AP, Tang WY, Fu DL, Liu Q, Sheng JK, Gu Z, Lee TY, Barkham T and Kyoung Park M: A rapid amplification/detection assay for analysis of Mycobacterium tuberculosis using an isothermal and silicon bio-photonic sensor complex. Biosens Bioelectron 68: 390-396, 2015. 
73. Krõlov K, Frolova J, Tudoran O, Suhorutsenko J, Lehto T, Sibul H, Mäger I, Laanpere M, Tulp I and Langel Ü: Sensitive and rapid detection of Chlamydia trachomatis by recombinase polymerase amplification directly from urine samples. J Mol Diagn 16: 127-135, 2014.

74. Clancy E, Higgins O, Forrest MS, Boo TW, Cormican M, Barry T, Piepenburg O and Smith TJ: Development of a rapid recombinase polymerase amplification assay for the detection of Streptococcus pneumoniae in whole blood. BMC Infect Dis 15: 481, 2015.

75. Mondal D, Ghosh P, Khan MA, Hossain F, Böhlken-Fascher S, Matlashewski G, Kroeger A, Olliaro P, Abd El and Wahed A: Mobile suitcase laboratory for rapid detection of Leishmania donovani using recombinase polymerase amplification assay. Parasit Vectors 9: 281, 2016.

76. Sriworarat C, Phumee A, Mungthin M, Leelayoova S and Siriyasatien P: Development of loop-mediated isothermal amplification (LAMP) for simple detection of Leishmania infection. Parasit Vectors 8: 591, 2015.

77. Nzelu CO, Cáceres AG, Guerrero-Quincho S, Tineo-Villafuerte E, Rodriquez-Delfin L, MimoriT, UezatoH, Katakura K, GomezEA, Guevara AG, et al: A rapid molecular diagnosis of cutaneous leishmaniasis by colorimetric malachite green-loop-mediated isothermal amplification (LAMP) combined with an FTA card as a direct sampling tool. Acta Trop 153: 116-119, 2016.

78. Jauset-Rubio M, Tomaso H, El-Shahawi MS, Bashammakh AS, Al-Youbi AO and O'Sullivan CK: Duplex lateral flow assay for the simultaneous detection of yersinia pestis and francisella tularensis. Anal Chem 90: 12745-12751, 2018.

79. Toldrà A, Jauset-Rubio M, Andree KB, Fernández-Tejedor M, Diogène J, Katakis I, O'Sullivan CK and Campàs M: Detection and quantification of the toxic marine microalgae karlodinium veneficum and karlodinium armiger using recombinase polymerase amplification and enzyme-linked oligonucleotide assay Anal Chim Acta 1039: 140-148, 2018

80. Al-Madhagi S, Joda H, Jauset-Rubio M, Ortiz M, Katakis I and O'Sullivan CK: Isothermal amplification using modified primers for rapid electrochemical analysis of coeliac disease associated DQB1*02 HLA allele. Anal Biochem 556: 16-22, 2018.

81. Sabaté del Río J, Steylaerts T, Henry OYF, Bienstman P, Stakenborg T, Van Roy W and O'Sullivan CK: Real-time and label-free ring-resonator monitoring of solid-phase recombinase polymerase amplification. Biosens Bioelectron 73: 130-137, 2015.

82. Wiedenheft B, Sternberg SH and Doudna JA: RNA-guided genetic silencing systems in bacteria and archaea. Nature 482 331-338, 2012.
83. Gootenberg JS, Abudayyeh OO, Lee JW, Essletzbichler P, Dy AJ, Joung J, Verdine V, Donghia N, Daringer NM, Freije CA, et al: Nucleic acid detection with CRISPR-Cas13a/C2c2. Science 356: 438-442, 2017.

84. Gootenberg JS, Abudayyeh OO, Kellner MJ, Joung J, Collins JJ and Zhang F: Multiplexed and portable nucleic acid detection platform with Cas13, Cas12a, and Csm6. Science 360: 439-444, 2018.

85. Chen JS, Ma E, Harrington LB, Da Costa M, Tian X, Palefsky JM and Doudna JA: CRISPR-Cas12a target binding unleashes indiscriminate single-stranded DNase activity. Science 360: 436-439, 2018.

86. Chakrabarti R and Schutt CE: The enhancement of PCR amplification by low molecular weight amides. Nucleic Acids Res 29: 2377-2381, 2001

87. Kovárová M and Dráber P: New specificity and yield enhancer of polymerase chain reactions. Nucleic Acids Res 28: E70, 2000.

88. Chester N and Marshak DR: Dimethyl sulfoxide-mediated primer Tm reduction: A method for analyzing the role of renaturation temperature in the polymerase chain reaction. Anal Biochem 209: 284-290, 1993.

89. Sarkar G, Kapelner S and Sommer SS: Formamide can dramatically improve the specificity of PCR. Nucleic Acids Res 18: 7465, 1990.

90. Yasukawa K, Konishi A and Inouye K: Effects of organic solvents on the reverse transcription reaction catalyzed by reverse transcriptases from avian myeloblastosis virus and Moloney murine leukemia virus. Biosci Biotechnol Biochem 74: 1925-1930, 2010.

91. Ahokas $\mathrm{H}$ and Erkkilä MJ: Interference of PCR amplification by the polyamines, spermine and spermidine. PCR Methods Appl 3: 65-68, 1993.

92. Roperch JP, Benzekri K, Mansour H and Incitti R: Improved amplification efficiency on stool samples by addition of spermidine and its use for non-invasive detection of colorectal cancer. BMC Biotechnol 15: 41, 2015.

93. Kikuchi A, Sawamura T, Kawase N, Kitajima Y, Yoshida T, Daimaru O, Nakakita T and Itoh S: Utility of spermidine in PCR amplification of stool samples. Biochem Genet 48: 428-432, 2010. 